\title{
Presence of both alterations in FGFR/FGF and PI3K/AKT/mTOR confer improved outcomes for patients with metastatic breast cancer treated with PI3K/AKT/mTOR inhibitors
}

\author{
Jennifer J. Wheler ${ }^{1}$, Johnique T. Atkins ${ }^{2}$, Filip Janku², Stacy L. Moulder ${ }^{3}$, Philip J. \\ Stephens ${ }^{4}$, Roman Yelensky, ${ }^{4}$ Vicente Valero ${ }^{3}$, Vincent Miller ${ }^{4}$, Razelle Kurzrock ${ }^{5}$, \\ Funda Meric-Bernstam² \\ ${ }^{1}$ Translational clinical oncology, Novartis Pharmaceuticals, Cambridge, MA, USA \\ ${ }^{2}$ Department of Investigational Cancer Therapeutics, The University of Texas MD Anderson Cancer Center, Houston, TX, USA \\ ${ }^{3}$ Department of Breast Medical Oncology, The University of Texas MD Anderson Cancer Center, Houston, TX, USA \\ ${ }^{4}$ Foundation Medicine, Cambridge, MA, USA \\ ${ }^{5}$ The Center for Personalized Cancer Therapy, University of California San Diego Moores Cancer Center, La Jolla, CA, USA \\ Correspondence to: Johnique T. Atkins, email: johniqueatkins@gmail.com \\ Keywords: breast cancer, FGFR, next-generation sequencing, PI3K \\ Received: March 22, 2016 \\ Accepted: May 13, 2016 \\ Published: June 30, 2016
}

\section{ABSTRACT}

There is limited data on co-expression of FGFR/FGR amplifications and PI3K/ AKT/mTOR alterations in breast cancer. Tumors from patients with metastatic breast cancer referred to our Phase I Program were analyzed by next generation sequencing (NGS). Genomic libraries were selected for all exons of 236 (or 182) cancer-related genes sequenced to average depth of $>500 \times$ in a CLIA laboratory (Foundation Medicine, Cambridge, MA, USA) and analyzed for all classes of genomic alterations. We report genomic profiles of 112 patients with metastatic breast cancer, median age 55 years (range, $27-78$ ). Twenty-four patients $(21 \%)$ had at least one amplified FGFR or FGF. Fifteen of the 24 patients $(63 \%)$ also had an alteration in the PI3K/ AKT/mTOR pathway. There was no association between alterations in FGFR/FGF and PI3K/AKT/mTOR $(P=0.49)$. Patients with simultaneous amplification in FGFR/FGF signaling and the PI3K/AKT/mTOR pathway had a higher rate of SD $\geq 6$ months/PR/ CR when treated with therapies targeting the PI3K/AKT/mTOR pathway than patients with only alterations in the PI3K/AKT/mTOR pathway $(73 \%$ vs. $34 \% ; P=0.0376)$ and remained on treatment longer (6.8 vs. 3.7 months; $P=0.053$ ). Higher response rates were seen in patients with simultaneous amplification in FGFR/FGF signaling and alterations in the PI3K/AKT/mTOR pathway who were treated with inhibitors of that pathway.

\section{INTRODUCTION}

Fibroblast growth factor receptors (FGFR) and their ligands (FGF) play a critical role in proliferation, migration, angiogenesis, and survival of cancer cells. FGFR signaling has been primarily described as an escape mechanism of vascular endothelial growth factor (VEGF) targeted therapies, but recent studies have also identified alterations in FGFRs as driving oncogenes in certain tumor types $[1,2]$. Alterations in FGFR signaling include, but are not limited to, gene amplification or post-transcriptional regulation leading to receptor overexpression; FGFR mutations producing receptors that are either constitutively active or have a reduced dependence on ligand binding for activation; and upregulation of FGF expression and the enhanced release of FGFs from the extracellular matrix, resulting in paracrine/autocrine activation of the pathway [3]. Dysregulation of FGFR signaling can lead to downstream activation of mitogen activated protein kinase (MAPK) and phosphoinositide-3-kinase (PI3K)/ 
AKT pathways $[2,4,5]$. FGFR signaling also interacts with phospholipase C-gamma (PLC- $\gamma$ ) to stimulate protein kinase $\mathrm{C}$ (PKC), which phosphorylates RAF in the MAPK pathway [2].

There are four identified FGFR receptors, FGFR1, FGFR2, FGFR3, and FGFR4 that bind to a variety of FGFs. Twenty-two different FGFs have also been identified. FGF1 through $F G F 10$ all bind to FGFRs. $F G F 11$ through $F G F 14$ have similar homologies to the other FGFs, but do not bind to FGFRs and have distinct functional differences. $F G F 19, F G F 21$, and $F G F 23$ are considered hormone-like and, in contrast to the local activity of the other FGFs, have systemic effects. The different FGFs and their corresponding receptors are expressed in a tissue specific manner, contributing to the specificity of the ligand-receptor interaction $[2,6]$.

Members of the FGFR family are rarely mutated but frequently amplified or overexpressed in breast cancer, which is often accompanied by increase, or altered, expression of FGF ligands [7]. Hybrid capture based broad next-generation sequencing (NGS) has allowed us to take an in-depth look at the genomic landscape of breast cancer patients seen in our phase I clinic [8]. The purpose of this study was to estimate the frequency of alterations in FGFRs and FGFs and to characterize the nature of these alterations in a population of patients with advanced, heavily pretreated breast cancer. A secondary objective was to report on any associations between molecular profile and response to targeted therapy.

\section{RESULTS}

\section{Patients}

A total of 112 patients with advanced breast cancer had their tumors analyzed by Foundation Medicine either prospectively to determine an appropriate clinical trial with targeted therapy or retrospectively to correlate with response to therapy. Median age was 55 years (range, 27 to 78 years). Ninety patients $(80 \%)$ were white; nine ( $8 \%)$ were African American; ten (9\%) were Hispanic; and, four (3\%) were Asian. Fifty-five patients (49\%) were hormone receptor (HR)-positive (estrogen or progesterone) and eight $(7 \%)$ were HER2-positive. Detailed patient characteristics are listed in Table 1.

\section{FGFR/FGF amplification}

A complete list of FGFR and FGF amplifications is listed in Table 2. Of 112 patients, 24 (21\%) had at least one amplified FGFR or FGF. Twelve of the 24 patients $(50 \%)$ had more than one amplification. The presence of FGFR/FGF amplification was not significantly associated with age, ethnicity, hormone receptor status, HER2 status or site of mutation analysis (primary vs. metastatic tumor tissue).

The most common amplification was in FGFR1, seen in $8(7 \%)$ patients. Five of the eight (63\%) patients were hormone-receptor (estrogen and/or progesterone) positive. Three $(3 \%)$ patients had an amplification in $F G F R 2$, all of whom were triple-negative. FGFR3 amplification was seen one patient $(>1 \%)$ who was estrogen-receptor positive. FGFR4 amplification was not seen.

Amplification in FGF3, FGF4, and FGF19 appeared simultaneously in $10(9 \%)$ patients. Three patients had amplification in $F G F 23$, two patients had amplification in FGF6 and amplification in FGF10, and FGF14 were seen in one patient each.

\section{Simultaneous alterations}

Of the 24 patients with amplification in FGFR or FGF, $15(63 \%)$ also had an amplification in CCND1. All 10 patients with amplification in $F G F 3, F G F 4$ and $F G F 19$ also had amplification in CCND1. Fifteen patients (63\%) with an FGF/FGFR also had an alteration affecting the $\mathrm{PI} 3 \mathrm{~K} / \mathrm{AKT} / \mathrm{mTOR}$ pathway, including alterations in $P I K 3 C A, A K T, P T E N$ and NFI. There was not a statistical association between alterations in the PI3K/AKT/mTOR pathway and the presence of FGFR/FGF amplifications $(P=0.49)$. None of the patients with amplification in FGFR or FGF had amplification in HER2.

\section{Response to targeted therapies}

Three of the 112 patients in this study received a non-selective FGFR inhibitor, including two of the 24 patients (8\%) with FGFR or FGF amplification. Both of these patients had amplification of FGF3, FGF4 and $F G F 19$. A response was not seen with this treatment. One patient with FGFR3 amplification was treated with the tyrosine kinase inhibitor pazopanib, that has some FGFR activity, with no response seen. Eleven of the 15 patients with FGF/FGFR amplification and an alteration in the $\mathrm{PI} 3 \mathrm{~K} / \mathrm{AKT} / \mathrm{mTOR}$ pathway received therapy targeting the PI3K/AKT/mTOR pathway and were evaluable for a response. Eight of the eleven patients (73\%) experienced stable disease $(\mathrm{SD}) \geq 6$ months/partial response (PR)/ complete response (CR). In comparison, of 35 patients without FGF/FGFR amplification who had an alteration in the PI3K/AKT/mTOR pathway were treated with a therapy targeting this pathway and were evaluable for a response, 12 (34\%) experienced $\mathrm{SD} \geq 6$ months/PR/CR $(P=0.0376)$. The TTF for patients with both type of alterations was 6.8 months (95\% CI 2.413-11.187) compared to 3.7 months (95\% CI 2.39-5.01) patients with only alterations in the PI3K/AKT/mTOR pathway $(P=0.053)$. Seven patients with FGF/FGFR amplification and no alterations in the 
Table 1: Histopathologic and Molecular Characteristics of 24 Patients with Amplifications in FGFR/FGF signaling

\begin{tabular}{llllccc}
\hline Case No. Histology & ER status & PR status & HER2 status & $\begin{array}{c}\text { Biopsy } \\
\text { Location }\end{array}$ & Molecular Profile \\
\hline
\end{tabular}

ATM mutation R189K,

1 Ductal Positive Positive Negative Liver

2 Ductal Positive Positive Negative Breast

3 Metaplastic Negative Negative Negative Breast

deletion, FGFR2 amplification,

MYC amplification, TP53

mutation $\mathrm{R} 273 \mathrm{C}$

FGFR2 amplification, TP53

4 Ductal Negative Negative Negative Chest wall

mutation R306*, CDH1 mutation

$$
\text { Q264* }
$$

AKT1 mutation E17K,

KIT amplification, FGFR1

5 Ductal Positive Positive Negative Lymph node

amplification, MYC amplification,

TP53 mutation P36fs*7,

NFKBIA amplification,

BCL2L2 amplification, ZNF703

amplification

PIK3CA mutation H1047R,

PIK3R1 mutation G376R,

6 Ductal Positive Positive Negative Metastasis

CCND1 amplification, FGFR1

amplification, MYC amplification,

MCL1 amplification, FBXW7

mutation D112E

FGF14 amplification, GATA3

7 Ductal Negative Negative Negative Liver

mutation A333fs*20, IRS2

amplification

TSC2 mutation G1055fs*113,

FGFR1 amplification, ATM

8 Ductal Negative Negative Negative Breast

mutation V1569fs*29, MCL1

amplification, TP53 mutation

L111fs*40, MYC amplification,

MYST3 amplification

CDKN2A mutation Y44fs*1,

TP53 mutation, CCND1

amplification, CCND2

9 Ductal Negative Negative Negative Breast

amplification, FGF19

amplification, FGF3 amplification,

FGF4 amplification, FGF6 amplification

(Continued) 


\begin{tabular}{|c|c|c|c|c|c|c|}
\hline Case No. & Histology & ER status & PR status & HER2 status & $\begin{array}{c}\text { Biopsy } \\
\text { Location }\end{array}$ & Molecular Profile \\
\hline 10 & Ductal & Negative & Negative & Negative & Breast & $\begin{array}{c}\text { PTEN deletion, FGFR1 } \\
\text { amplification, CCNE1 } \\
\text { amplification, MCL1 } \\
\text { amplification, MYC amplification, } \\
\text { TP53 mutation Y234*, BCL2L2 } \\
\text { amplification, NPM1 truncation } \\
\text { 5'UTR, FAM123B mutation } \\
\text { G303D }\end{array}$ \\
\hline 11 & Ductal & Positive & Positive & Negative & Metastasis & $\begin{array}{l}\text { CCND1 amplification, FGFR1 } \\
\text { amplification, PRKDC } \\
\text { rearrangement, PTEN protein loss }\end{array}$ \\
\hline 12 & Ductal & Negative & Negative & Negative & Breast & $\begin{array}{c}\text { FGFR1 amplificaiton, FGFR2 } \\
\text { amplification, CCND1 } \\
\text { amplification, MAP2K2 } \\
\text { amplification, MYC amplification, } \\
\text { TP53 mutation C242fs*5, PTEN } \\
\text { protein loss }\end{array}$ \\
\hline 13 & Ductal & Positive & Positive & Negative & Breast & $\begin{array}{c}\text { CCND1 amplification, FGFR1 } \\
\text { amplification, ARID1A mutation } \\
\text { Q708* }\end{array}$ \\
\hline 14 & Metaplastic & Negative & Negative & Negative & Breast & $\begin{array}{l}\text { PIK3R1 mutation Y580fs*19, } \\
\text { CCND2 amplification, CDKN2A } \\
\text { deletion, FGF23 amplification }\end{array}$ \\
\hline 15 & Ductal & Positive & Negative & Negative & Liver & $\begin{array}{c}\text { PIK3CA mutation E454K, } \\
\text { BRCA2 mutation K3326*, } \\
\text { CCND1 amplification, HRAS } \\
\text { mutation G12D, GATA3 } \\
\text { mutation D336fs*17, FGF19 } \\
\text { amplification, FGF3 amplification, } \\
\text { FGF4 amplification, MYST3 } \\
\text { amplification }\end{array}$ \\
\hline 16 & Ductal & Positive & Positive & Negative & Breast & $\begin{array}{c}\text { PTEN mutation E7fs*9, BRCA2 } \\
\text { mutation K3326*, CCND1 } \\
\text { amplification, EP300 mutation } \\
\text { P925T, FGF19 amplification, } \\
\text { FGF3 amplification, FGF4 } \\
\text { amplification }\end{array}$ \\
\hline 17 & Ductal & Positive & Positive & Negative & Breast & $\begin{array}{c}\text { ERBB4 amplification, HGF } \\
\text { amplification, RICTOR } \\
\text { amplification BCL2L2 } \\
\text { amplification, JUN amplification, } \\
\text { FGF10 amplification }\end{array}$ \\
\hline 18 & Ductal & Positive & Negative & Negative & Abdomen & $\begin{array}{c}\text { FGFR3 amplification, PIK3CA } \\
\text { mutation H1047L, CCND1 } \\
\text { amplification, TP53 mutation } \\
\text { R249M }\end{array}$ \\
\hline
\end{tabular}




\begin{tabular}{|c|c|c|c|c|c|c|}
\hline Case No. & Histology & ER status & PR status & HER2 status & $\begin{array}{c}\text { Biopsy } \\
\text { Location }\end{array}$ & Molecular Profile \\
\hline 19 & Papillary & Negative & negative & Negative & Lymph node & $\begin{array}{c}\text { TP53 mutation S313fs*34, } \\
\text { CCND1 amplification, FGF19 } \\
\text { amplification, FGF3 amplification, } \\
\text { FGF4 amplification }\end{array}$ \\
\hline 20 & Ductal & Positive & Negative & Negative & Liver & $\begin{array}{c}\text { PIK3CA mutation E542K, } \\
\text { CCND1 amplification, } \\
\text { MCL1 amplification, FGF19 } \\
\text { amplification, FGF3 amplification, } \\
\text { FGF4 amplification, GATA3 } \\
\text { mutation I362fs*49+, MAP2K4 } \\
\text { mutation E299* }\end{array}$ \\
\hline 21 & Ductal & Positive & Not Known & Negative & Breast & $\begin{array}{c}\text { PIK3CA mutation E545K, } \\
\text { PIK3CA mutation Q546H, ATM } \\
\text { mutation R2832C, CCND1 } \\
\text { amplification, ARID1A mutation } \\
\text { R1528*, FGF19 amplification, } \\
\text { FGF3 amplification, FGF4 } \\
\text { amplification }\end{array}$ \\
\hline 22 & Ductal & Positive & Negative & Negative & Breast & $\begin{array}{l}\text { PTEN loss, KRAS amplification, } \\
\text { MYC amplification, CCND2 } \\
\text { amplification, TP53 mutation } \\
\text { V272M, RB1 loss, FGF23 } \\
\text { amplification, FGF6 amplification, } \\
\text { KDM5A amplification }\end{array}$ \\
\hline 23 & Ductal & Positive & Positive & Negative & Breast & $\begin{array}{l}\text { PTEN splice } 1087 \text { 1088ins47+, } \\
\text { TSC2 mutation N363fs*29, } \\
\text { FGFR1 amplification, } \\
\text { CCND1 amplification, } \\
\text { MDM2 amplification, MYC } \\
\text { amplification, TP53 mutation } \\
\text { P278T, MDM4 amplification, } \\
\text { FGF19 amplification, FGF3 } \\
\text { amplification, FGF4 amplification, } \\
\text { MYST3 amplification, ZNF703 } \\
\text { amplification }\end{array}$ \\
\hline 24 & Ductal & Positive & Negative & Negative & Breast & $\begin{array}{c}\text { CCND1 amplification, Ep300 } \\
\text { mutation P925T, FGF19 } \\
\text { amplification, FGF4 amplification }\end{array}$ \\
\hline
\end{tabular}

$\mathrm{PI} 3 \mathrm{~K} / \mathrm{AKT} / \mathrm{mTOR}$ pathway received an inhibitor of this pathway and were evaluable for a response and one (14\%) experienced $\mathrm{SD} \geq 6$ months/PR/CR.

\section{DISCUSSION}

In our study, we observed FGFR and FGF amplification in $21 \%$ of patients with metastatic breast cancer who underwent NGS profiling. No mutations or fusions in FGFR were seen, consistent with previous reports that these classes of alterations are rare in breast cancer. [7] FGFR and FGF amplification, however, were common in our patient population, with $21 \%$ of patients demonstrating amplification.

The most common amplification was in FGFR1, observed in $7 \%$ of patients. It has been previously reported that FGFR1 amplifications occur predominately in HRpositive patients [9], however; we observed similar rates 
Table 2: FGF and FGFR amplifications identified in 112 patients with metastatic breast cancer

\begin{tabular}{lc}
\hline Gene & \# of patients with amplification (\% of all patients) \\
\hline FGFR1 & $8(7 \%)$ \\
FGFR2 & $3(3 \%)$ \\
FGFR3 & $1(<1 \%)$ \\
FGF3 & $10(9 \%)$ \\
FGF4 & $10(9 \%)$ \\
FGF6 & $2(2 \%)$ \\
FGF10 & $1(<1 \%)$ \\
FGF14 & $1(<1 \%)$ \\
FGF19 & $10(9 \%)$ \\
FGF23 & $3(3 \%)$ \\
\hline
\end{tabular}

of FGFR 1 amplifications in HR-positive and HR-negative patients (9\% of HR-positive patients had an FGFRl amplification and 5\% of HR-negative patients had an FGFRl amplification). This may have been attributable in part to our small study size.

Our data is consistent with previous reports demonstrating the co-existence of amplifications in the 11q12-14 amplicon. This amplicon contains FGF3, FGF4, $F G F 19$, and $C C N D 1$. The simultaneous amplifications in FGF3, FGF4, FGF19, and CCND1 have been previously reported [10]. In our analysis 10 of 112 patients demonstrated amplification in FGF3, FGF4, FGF19 and $C C N D 1$. An additional patient had these amplifications as well had an amplification in FGFR1. We observed simultaneous amplification in FGFR1 and CCND1 in five of eight patients with an FGFR1 amplification [11].

We observed FGFR2 amplification exclusively in patients with triple-negative breast cancer ( 3 patients), consistent with previous reports [2, 12]. FGFR3 and FGFR4 amplification are less common than FGFR1 and $F G F R 2$ in breast cancer [3, 13]. Consistent with these reports, we observed one $F G F R 3$ amplification among all patients, in a patient who with HR-positive breast cancer. We did not observe FGFR4 amplification.

We observed that patients with simultaneous amplification in FGFR/FGF and alterations in the PI3K/ $\mathrm{AKT} / \mathrm{mTOR}$ pathway had a higher rate of $\mathrm{SD} \geq 6$ months/ $\mathrm{PR} / \mathrm{CR}$ and TTF when treated with therapies targeting the $\mathrm{PI} 3 \mathrm{~K} / \mathrm{AKT} / \mathrm{mTOR}$ pathway than patients with alterations in the PI3K/AKT/mTOR pathway. This difference was statistically significant ( $73 \%$ vs. $34 \% ; P=0.0376)$. Since FGFR/FGF signaling is known to activate the PI3K/AKT/ mTOR pathway (Figure 1) [14], tumors with simultaneous alterations may be more dependent on or "addicted" to this pathway for growth and survival, making it an attractive target for tumors with both of these types of alterations.
Previous studies have shown that activation of $A K T$ is at least partially responsible for resistance to FGFR inhibitors in mammary and gastric cell lines with amplified FGFR levels $[15,16]$. It was also determined that the combination of an FGFR inhibitor with rapamycin (a mTOR inhibitor) enhances the anti-proliferative effects in FGFR-addicted cells, suggesting that the combination of FGFR inhibitors with inhibitors of the $\mathrm{PI} 3 \mathrm{~K} / \mathrm{AKT} / \mathrm{mTOR}$ pathway may be an effective strategy for clinical development [15]. Notably, the two patients in our analysis who experienced clinical progression while receiving a nonselective FGFR inhibitor also had activating mutations in $P I K 3 C A$. We hypothesize that a lack of a clinical response in these patients could be due to an overactive PI3K/AKT/mTOR pathway. Only $14 \%$ of the patients ( 1 of 7) with an FGF/FGFR amplification, but no PI3K/AKT/mTOR alteration, who were treated with an inhibitor of the PI3K/AKT/mTOR pathway experienced $\mathrm{SD} \geq 6$ months/PR/CR, which suggests therapy targeting this pathway may not be effective in tumors with in FGF/ FGFR alone.

This study has several limitations, including its small sample size. In addition, at the time these patients were treated there was limited availability of FGFRdirected therapies. Only four patients were treated with FGFR inhibitors. Another limitation is that this study represents retrospective data. Unfortunately, results of the hybrid capture based NGS were typically not used in selection of targeted therapies. A prospective study allowing for selection of matched therapies would more accurately reflect associations between these molecular alterations and response. Genomic profiling was performed on available tissue that was either from the primary tumor or a metastatic site. Ideally, both primary and metastatic tissue would be analyzed with the approach described herein. Pre- and post-treatment biopsies are also 
helpful to identify changes in molecular profile that occur over time and in response to therapy.

Despite the limitations of this study, our data suggests that concomitant presence of FGFR/FGF amplification and alterations in the setting of an activated $\mathrm{PI} 3 \mathrm{~K} / \mathrm{AKT} / \mathrm{mTOR}$ pathway may predict for better outcomes to $\mathrm{PI} 3 \mathrm{~K} / \mathrm{AKT} / \mathrm{mTOR}$ inhibitors. Given the prevalence of $\mathrm{PI} 3 \mathrm{~K} / \mathrm{AKT} / \mathrm{mTOR}$ pathway alterations in patients with breast cancer [17-19] and the availability of several inhibitors of this pathway, this coexistence of molecular alterations may be an important biomarker. It is also suggestive that combination approaches to treatment that include both an FGFR inhibitor and a PI3K/AKT/ mTOR inhibitor may be beneficial. Further studies in larger groups of patients are ongoing.

\section{PATIENTS AND METHODS}

\section{Patients}

Patients with advanced and metastatic breast cancers who experienced treatment failure with standard therapy and who had tissue available for mutation analysis were eligible. The study was conducted in the Department of Investigational Cancer Therapeutics (Phase I Clinical Trials Program) at MD Anderson Cancer Center. The registration of patients in the database and pathology assessment was performed at MD Anderson. Hybrid capture based, comprehensive next-generation sequencing of tumor tissue was performed at Foundation Medicine using FoundationOne ${ }^{\mathrm{TM}}$ (Cambridge, MA). Eligible

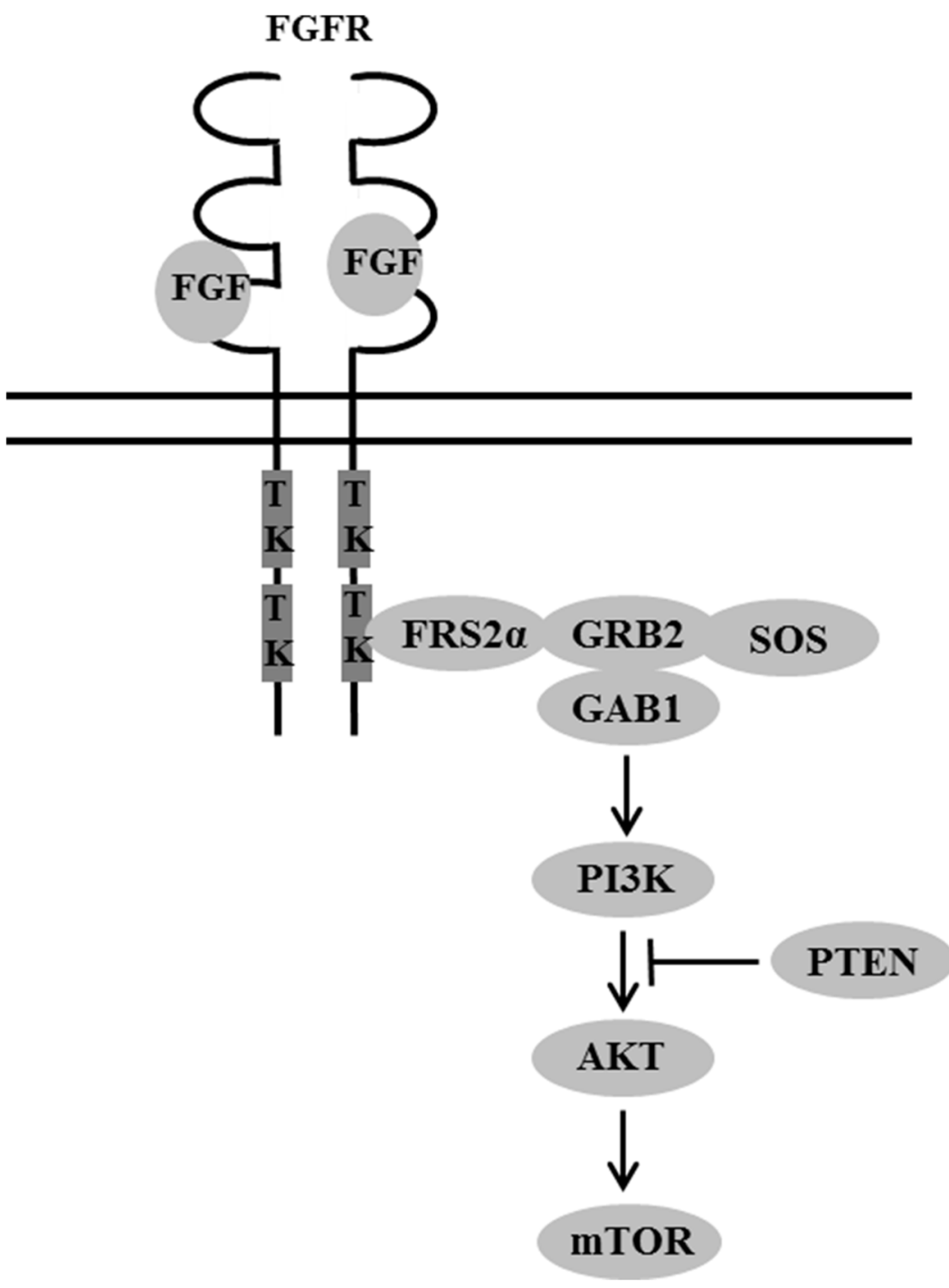

Figure 1: FGFR interaction with PI3K/AKT/mTOR pathway. TK, tyrosine kinase domains; FRS2 $\alpha$, fibroblast growth factor receptor substrate $2 \alpha$. 
patients were those referred for phase I clinical trials for targeted therapeutic agents. This study and all treatments were conducted in accordance with the guidelines of the MD Anderson Institutional Review Board. Some patients had their tumors analyzed prospectively and were enrolled on trials according to molecular profile, while others had their tumors analyzed retrospectively after already being enrolled on a trial.

\section{Evaluation of HER2 amplification, estrogen and progesterone receptor status, PTEN protein loss}

Under CLIA conditions, immunohistochemistry was used to measure of HER2, estrogen and progesterone receptors and the presence of PTEN protein loss. Estrogen and progesterone receptors were assessed using antibody 6F11 (Novocastra Laboratories, Ltd., Newcastle Upon Tyne, UK). Alternatively, fluorescence in situ hybridization (FISH) was used to measure the copy number of HER2 according to current guidelines.

\section{Hybrid captured based comprehensive next- generation sequencing}

Tumor samples were evaluated for genomic alterations including base substitutions, short insertions and deletions, amplifications, homozygous deletions, gene fusions, truncations and rearrangements (Foundation Medicine, Cambridge, MA). DNA was extracted from $40 \mu \mathrm{m}$ of FFPE tissue (minimum $20 \%$ tumor cells) using the Maxwell 16 FFPE Plus LEV DNA Purification kit (Promega) and quantified using a standardized PicoGreen fluorescence assay (Invitrogen). Library Construction was performed using 50-200 ng of DNA sheared by sonication to $\sim 100-400$ bp before end-repair, dA addition and ligation of indexed, Illumina sequencing adaptors. Enrichment of target sequences (all coding exons of 182 or 236 cancer-related genes and selected introns from 14 or 19 genes recurrently rearranged in cancer) was achieved by solution-based hybrid capture with custom biotinylated oligonucleotide baits. Enriched libraries were sequenced to an average median depth of $>500 \mathrm{X}$ with $99 \%$ of bases covered $>100 X$ (Illumina HiSeq 2000 platform using $49 \times$ 49 paired-end reads) and mapped to the reference human genome (hg19) using the Burrows-Wheeler Aligner and the publicly available SAMtools, Picard and Genome Analysis Toolkit. Point mutations were identified by a Bayesian algorithm; short insertions and deletions, determined by local assembly; gene copy number alterations (amplifications), by comparison to process matched normal controls; and gene fusions/ rearrangements, by clustering chimeric reads mapped to targeted introns. Local site permissions to use clinical samples were also obtained. Genes were considered amplified if the copy number was amplified 5 times for ERBB2 (HER2) and 6 times for all other genes.

\section{Treatment and evaluation}

Assignment to a clinical trial was determined after clinical, laboratory, and pathologic data from all available patient records were reviewed. Consecutive patients who had tumor tissue that could be tested or had been tested with underlying alterations were enrolled, whenever possible, in clinical trials that directly targeted that alteration. Some patients, whose tumors were analyzed for alterations retrospectively, were also enrolled in clinical trials without such knowledge. Treatment continued until disease progression or unacceptable toxicity occurred. Treatment was carried out according to the specific requisites in the treatment protocols selected.

Assessments, including history, physical examination, and laboratory evaluations, were performed as specified in each protocol, typically before the initiation of therapy, weekly during the first cycle, and then, at a minimum, at the beginning of each new treatment cycle. Efficacy was assessed using computed tomography scans and/or magnetic resonance imaging at baseline before treatment initiation and then every two cycles (6 to 8 weeks). All radiographs were read in the Department of Investigational Cancer Therapeutics tumor measurement clinic. Responses were categorized per Response Evaluation Criteria in Solid Tumors (RECIST) and were reported as best response [20]. In brief, complete response (CR) was defined as the disappearance of all measurable and nonmeasurable disease. Partial response (PR) was defined as at least a $30 \%$ decrease in the sum of the longest diameter of measurable target lesions. Progressive disease (PD) was defined as at least a $20 \%$ increase in the sum of the longest diameter of measurable target lesions, unequivocal progression of a nontarget lesion, or the appearance of a new lesion. Stable disease (SD) was defined as neither sufficient shrinkage to qualify for PR nor sufficient increase to qualify for PD. A confirmation of $\mathrm{CR} / \mathrm{PR}$ required repeat imaging at least 28 days after the initial response assessment.

\section{Statistical analysis}

Two-way contingency tables were formed to summarize the relationship between two categorical variables. The Fisher's exact test was used to assess the association among categorical variables and alteration status. Time to treatment failure (TTF) was defined as the time interval from the start of therapy to the termination of treatment for any reason, including withdrawal of patient consent, uncontrolled toxicities, disease progression or death. Patients still receiving treatment at the time of 
analysis were censored at the last follow-up date. Median TTF was estimated using the method of Kaplan and Meier and were compared among subgroups of patients using a log-rank test. All tests were two-sided, and $P<0.05$ was considered statistically significant. All statistical analyses were carried out using SPSS 17 software (SPSS, Chicago, IN, USA).

\section{CONFLICTS OF INTEREST}

The authors declare no conflicts of interest.

\section{REFERENCES}

1. Crawford Y, Ferrara N. Tumor and stromal pathways mediating refractoriness/resistance to anti-angiogenic therapies. Trends Pharmacol Sci. 2009; 30:624-630.

2. Turner N, Grose R. Fibroblast growth factor signalling: from development to cancer. Nat Rev Cancer. 2010; 10:116-129.

3. Dienstmann R, Rodon J, Prat A, Perez-Garcia J, Adamo B, Felip E, Cortes J, Iafrate AJ, Nuciforo P, Tabernero J. Genomic aberrations in the FGFR pathway: opportunities for targeted therapies in solid tumors. Ann Oncol. 2014; 25:552-563.

4. Acevedo VD, Ittmann M, Spencer DM. Paths of FGFRdriven tumorigenesis. Cell Cycle. 2009; 8:580-588.

5. Tenhagen M, van Diest PJ, Ivanova IA, van der Wall E, van der Groep P. Fibroblast growth factor receptors in breast cancer: expression, downstream effects, and possible drug targets. Endocr Relat Cancer. 2012; 19:R115-129.

6. Eswarakumar VP, Lax I, Schlessinger J. Cellular signaling by fibroblast growth factor receptors. Cytokine Growth Factor Rev. 2005; 16:139-149.

7. Cancer GAN. Comprehensive molecular characterization of human breast tumors. Nature. 2012; 490:61-70.

8. Meric-Bernstam F, Frampton GM, Ferrer-Lozano J, Yelensky R, Perez-Fidalgo JA, Wang Y, Palmer GA, Ross JS, Miller VA, Su X, Eroles P, Barrera JA, Burgues O, Lluch AM, Zheng X, Sahin A, et al. Concordance of genomic alterations between primary and recurrent breast cancer. Mol Cancer Ther. 2014; 13:1382-1389.

9. Turner N, Pearson A, Sharpe R, Lambros M, Geyer F, Lopez-Garcia MA, Natrajan R, Marchio C, Iorns E, Mackay A, Gillett C, Grigoriadis A, Tutt A, Reis-Filho JS, Ashworth A. FGFR1 amplification drives endocrine therapy resistance and is a therapeutic target in breast cancer. Cancer Res. 2010; 70:2085-2094.

10. Kwek SS, Roy R, Zhou H, Climent J, Martinez-Climent JA, Fridlyand J and Albertson DG. Co-amplified genes at $8 \mathrm{p} 12$ and $11 \mathrm{q} 13$ in breast tumors cooperate with two major pathways in oncogenesis. Oncogene. 2009; 28:1892-1903.
11. Elbauomy Elsheikh S, AR G, MB L. FGFR1 amplification in breast carcinomas in situ hybridisation analysis. Breast Cancer Research. 2007; 9:R23.

12. Turner N, Lambros MB, Horlings HM, Pearson A, Sharpe R, Natrajan R, Geyer FC, van Kouwenhove M, Kreike B, Mackay A, Ashworth A, van de Vijver MJ, Reis-Filho JS. Integrative molecular profiling of triple negative breast cancers identifies amplicon drivers and potential therapeutic targets. Oncogene. 2010; 29:2013-2023.

13. Forbes SA, Bindal N, Bamford S, Cole C, Kok CY, Beare D, Jia M, Shepherd R, Leung K, Menzies A, Teague JW, Campbell PJ, Stratton MR, Futreal PA. COSMIC: mining complete cancer genomes in the Catalogue of Somatic Mutations in Cancer. Nucleic Acids Res. 2011; 39:D945-950.

14. Zhang L, Kharbanda S, Hanfelt J, Kern FG. Both autocrine and paracrine effects of transfected acidic fibroblast growth factor are involved in the estrogen-independent and antiestrogen-resistant growth of MCF-7 breast cancer cells. Cancer Res. 1998; 58:352-361.

15. $\mathrm{Hu} \mathrm{Y}, \mathrm{Lu} \mathrm{H}$, Zhang J, Chen J, Chai Z. Essential role of AKT in tumor cells addicted to FGFR. Anticancer Drugs. 2014; 25:183-188.

16. Dey JH, Bianchi F, Voshol J, Bonenfant D, Oakeley EJ, Hynes NE. Targeting fibroblast growth factor receptors blocks PI3K/AKT signaling, induces apoptosis, and impairs mammary tumor outgrowth and metastasis. Cancer Res. 2010; 70:4151-4162.

17. Janku F, Hong DS, Fu S, Piha-Paul SA, Naing A, Falchook GS, Tsimberidou AM, Stepanek VM, Moulder SL, Lee JJ, Luthra R, Zinner RG, Broaddus RR, Wheler JJ, Kurzrock R. Assessing PIK3CA and PTEN in early-phase trials with PI3K/AKT/mTOR inhibitors. Cell Rep. 2014; 6:377-387.

18. Janku F, Wheler JJ, Westin SN, Moulder SL, Naing A, Tsimberidou AM, Fu S, Falchook GS, Hong DS, GarridoLaguna I, Luthra R, Lee JJ, Lu KH, Kurzrock R. PI3K/ $\mathrm{AKT} / \mathrm{mTOR}$ inhibitors in patients with breast and gynecologic malignancies harboring PIK3CA mutations. J Clin Oncol. 2012; 30:777-782.

19. Stemke-Hale K, Gonzalez-Angulo AM, Lluch A, Neve RM, Kuo WL, Davies M, Carey M, Hu Z, Guan Y, Sahin A, Symmans WF, Pusztai L, Nolden LK, Horlings H, Berns $\mathrm{K}$, Hung $\mathrm{MC}$, et al. An integrative genomic and proteomic analysis of PIK3CA, PTEN, and AKT mutations in breast cancer. Cancer Res. 2008; 68:6084-6091.

20. Therasse P, Arbuck SG, Eisenhauer EA, Wanders J, Kaplan RS, Rubinstein L, Verweij J, Van Glabbeke M, van Oosterom AT, Christian MC, Gwyther SG. New guidelines to evaluate the response to treatment in solid tumors. European Organization for Research and Treatment of Cancer, National Cancer Institute of the United States, National Cancer Institute of Canada. J Natl Cancer Inst. 2000; 92:205-216. 